\title{
Common Lawn and Garden Mushrooms of Central Oklahoma
}

\author{
Clark L. Ovrebo \\ Department of Biology \\ University of Central Oklahoma \\ Edmond, OK 73034
}

Mushrooms are often abundant in lawns and gardens after periods of wet weather. This article presents photographs of some of the more common mushrooms the author has encountered in Central Oklahoma over the past fifteen years.

\section{INTRODUCTION}

A mushroom is the fruiting body of a fungus. The body of the fungus, termed mycelium, is found in soil, compost, wood chips, or logs. Rarely are mushroom species parasitic. The mycelium gets nutrition from breaking down the organic substrates (saprotrophic mode of nutrition). If the yard has oaks, hickories or pines, the fungus forms a mycorrhizal association with the tree roots, an association that is beneficial to both the fungus and the tree. Mycorrhizal fungi are most often found in forested areas, but may also occur in yards or city parks if their symbiotic trees are present. With the exception of Suillus brevipes, the fungi treated in this paper are saprotrophic.

The term "common," is not so easily defined. Some mushrooms appear every year after almost every rain, whereas others may appear only sporadically even after wet weather. Some fungi are abundant some years and not the next. Thus, the term "common" as used for fleshy fungi must remain vague. The time of year given for fruiting is also somewhat general and is intended to give an approximation of when the mushrooms fruit.

In addition to photos, a brief description is provided for each species. The intent is not to provide complete macroscopic and microscopic descriptions of the fungi, but rather, the salient morphological features. To be sure of identification, it may be necessary to use microscopic examination and consult field guides or technical literature. Spore print color is presented for some species because determining spore color is often the first step in identifying a mushroom. A spore print is made by cutting off the stipe and placing the pileus with lamellae side down on a piece of white paper. This set-up is covered and left overnight.

Advice is not provided about which lawn mushrooms are edible. Great care must be taken to be absolutely sure of a mushroom's identity. Only after becoming sufficiently familiar with mushroom characters and their identifying features can one be certain of the identification, and only then can the determination about edibility be made.

Many field guides are helpful and provide additional information. A listing of some field guides that are useful for Oklahoma is found at the end of the article.

\section{Agaricales}

Fungi in this order comprise one of the more common groups of lawn fungi and include what are called "gilled mushrooms" and "boletes". The parts of a gilled mushroom are the pileus (cap), stipe (stem), and lamellae (gills). A bolete has tubes that end in pores instead of lamellae. The spores are produced on the lamellae or tubes, forcibly discharged, and are dispersed by air currents.

Ovrebo, C.L.

https://doi.org/10.22488/okstate.17.100032 


\section{Chlorophyllum molybdites}

Scattered, on lawns or pastures, sometimes in fairy rings or arcs (Figure 1), late spring through mid-fall. Chlorophyllum molybdites is one of the most common larger mushrooms occurring on lawns. It is recognized by the rather large fruiting bodies, whitish pileus surface with tan scales near or on the center, green lamellae and by the partial veil which leaves an annulus on the stipe (Figure 2). The lamellae are off-white when young and become green at maturity, and are free (not attached to the stipe). The spore deposit is green. Chlorophyllum molybdites is the leading cause of mushroom poisoning in the United States. It causes severe gastrointestinal upset with recovery after several hours. It is especially toxic to young children and people already compromised by health problems.

\section{Amanita thiersii}

Scattered, on lawns, sometimes in fairy rings or arcs (Figure 3), summer and fall. Amanita thiersii is another of the larger mushrooms occurring on lawns and may be in fruit at the same time as C.molybdites. The pileus and stipe are white and the lamellae light cream-colored. Young fruiting bodies are covered with a flocculent coating that may remain throughout maturation (Figure 4). The covering is easily removed when touched and may be washed away with rain. The spore print is white. This species belongs in the genus that contains some of the most deadly poisonous mushrooms. Though little is known about the toxicity or edibility of $A$. thiersii, it is probably poisonous.

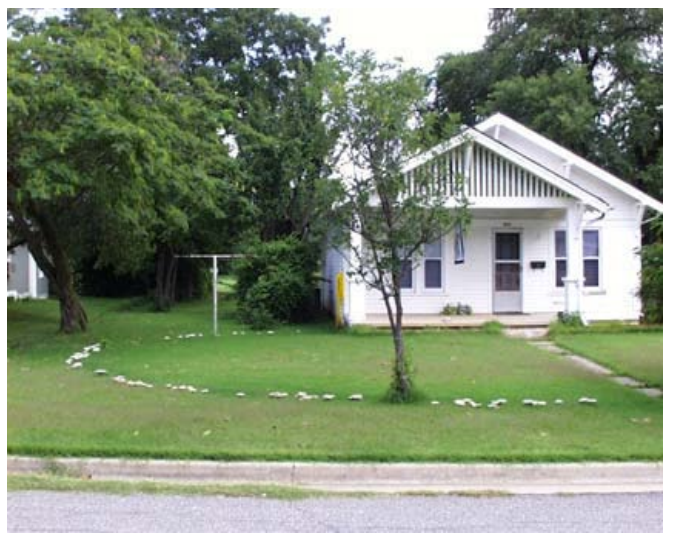

Figure 1 Chlorophyllum molybdites, fairy ring.

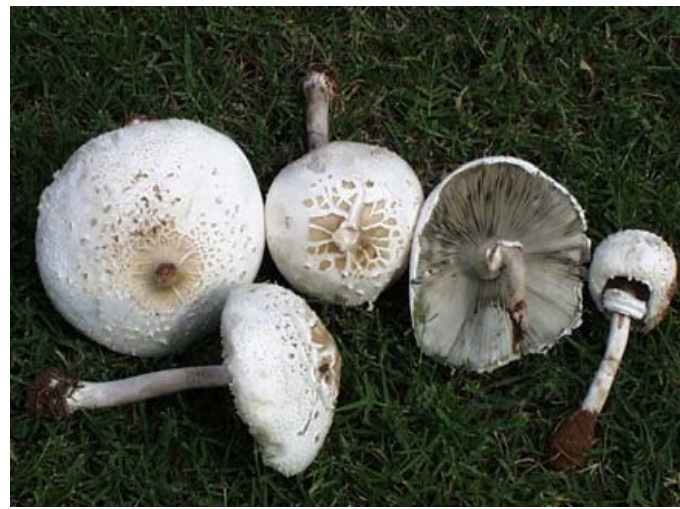

Figure 2 Chlorophyllum molybdites, fruiting bodies.

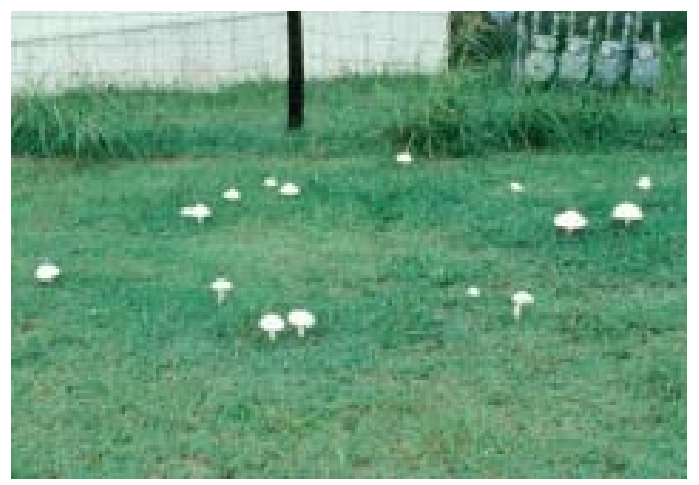

Figure 3 Amanita thiersii, fairy ring.

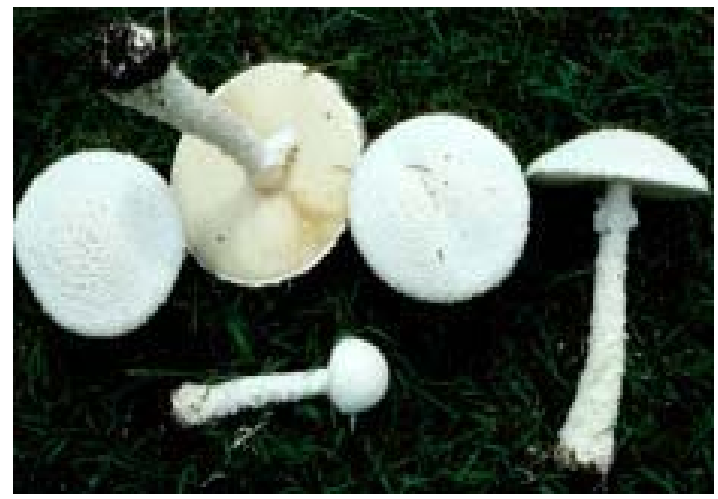

Figure 4 Amanita thiersii, fruiting bodies. 
Marasmius oreades (fairy ring mushroom)

Scattered, on lawns, occasionally in arcs or fairy rings (Figure 5), summer to late fall. Marasmius oreades is a small, thin-statured mushroom with the pileus reaching at most 3-4 $\mathrm{cm}$ in diameter. The pileus is smooth, off-white with the center often light $\tan$ (Figure 6). The buttons can be light brown overall. The lamellae are rather distantly spaced and off-white. The stipe is also off-white and lacks an annulus. The spore print is white.

\section{Coprinus comatus (shaggy mane)}

Scattered, on lawns, late summer through late fall. Coprinus comatus is easily recognized by the rather tall fruiting bodies that have a vertically elongated pileus. The pileus of the buttons is elliptical. The pileus has a shaggy surface and is off-white although the top can be $\tan$ (Figures 7 and 8). The lamellae are off-white when young and black at maturity. The genus is characterized by the fact that the mushrooms deliquesce (auto-digest). Beginning at the margin, the pileus begins to liquefy and the process continues toward the top-center of the pileus (Figure 9). Often only the stipe remains.

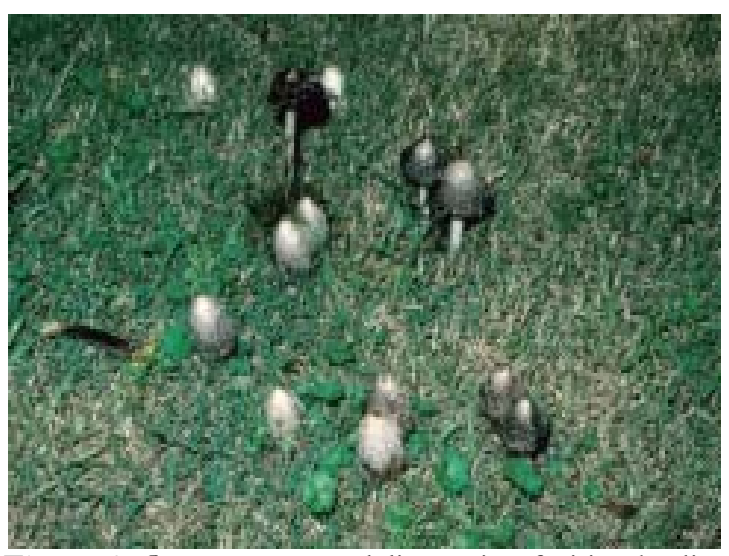

Figure 9 Coprinus comatus, deliquescing fruiting bodies

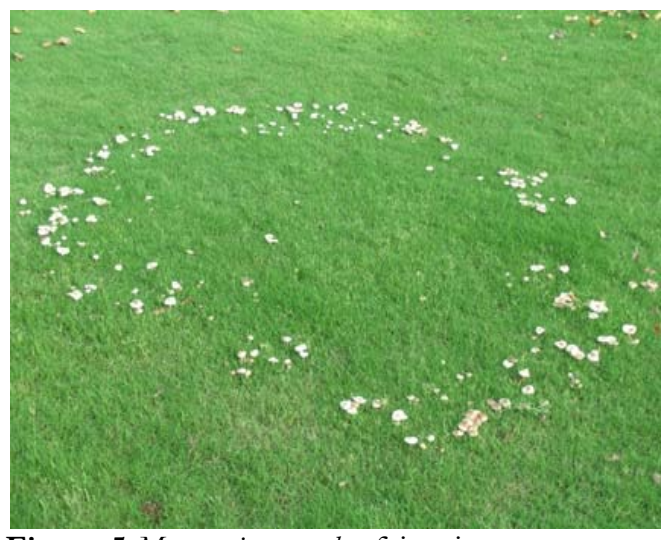

Figure 5 Marasmius oreades, fairy ring.

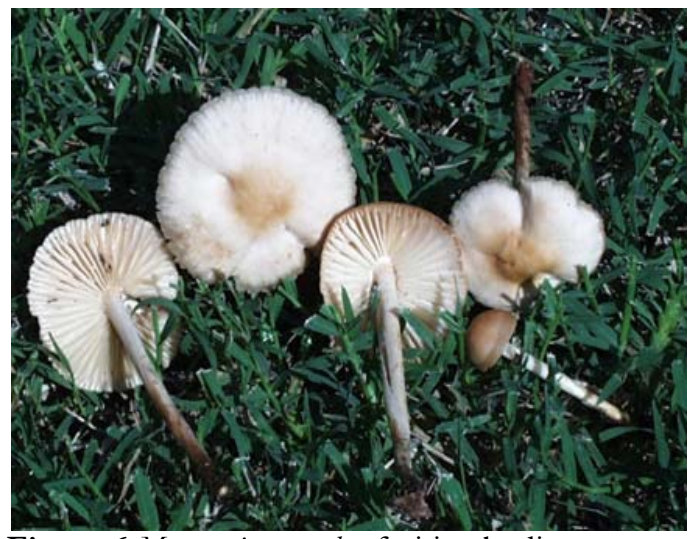

Figure 6 Marasmius oreades, fruiting bodies.

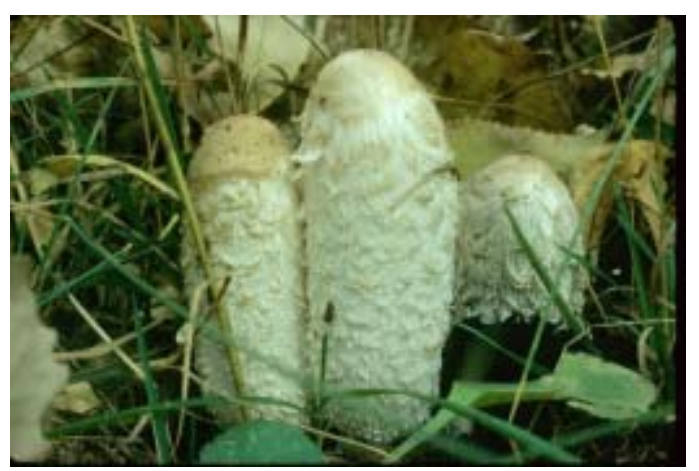

Figure 7 Coprinus comatus, fruiting bodies showing shaggy surface.

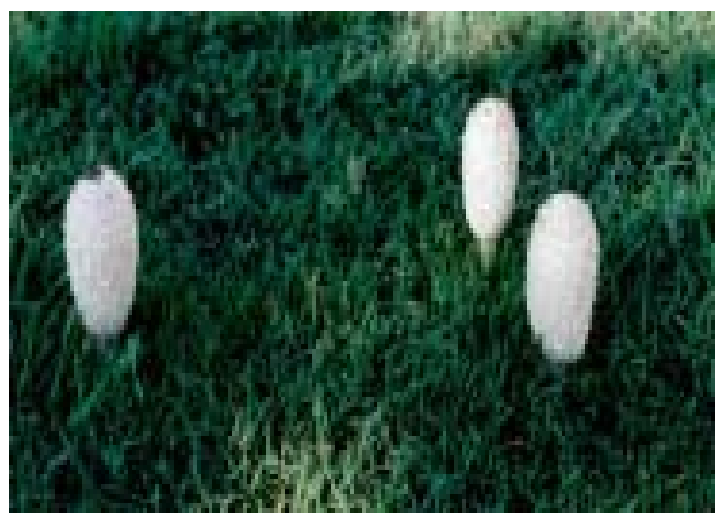

Figure 8 Coprinus comatus, mature fruiting bodies.

Ovrebo, C.L. 


\section{Coprinopsis variegata}

Scattered to clustered, on lawns, but attached to buried wood, or on stumps, summer. Coprinopsis variegata appears in clusters on lawns but is actually growing from buried wood such as the remains of a stump. The pileus is off-white to grayish brown to gray, and has scales or patches (Figures 10 and 11). Like Coprinus comatus, the fruiting bodies deliquesce. This species was formerly placed in Coprinus.

\section{Conocybe lactea}

Scattered, on lawns, early summer. Conocybe lactea has small fragile fruiting bodies that are evident in the morning but wither away as the heat of the day sets in. The pileus is whitish to light tan and rounded-conic. The lamellae are light cinnamon-brown (Figure 12).

\section{Parasola plicatilis}

Scattered, on lawns, early summer. Fruiting bodies of Parasola plicatilis are small and thin-statured (Figure 13). The pileus is translucent-gray and plicate (grooved). The lamellae are black. This species is most noticeable in the morning. In sunlight it quickly dries and disappears. This species was also formerly placed in Coprinus.

\section{Agaricus campestris (meadow mushroom)}

Scattered, on lawns and pastures, summer to early fall. Agaricus campestris is characterized by the whitish pileus and stipe, annulate stipe and by the dark brown, free lamellae (Figure 14). The lamellae start out light pink in the button stage and become dark brown as the spores mature. The spore print is dark brown. This species is in the same genus as the cultivated button mushroom that is available fresh or canned in grocery stores.

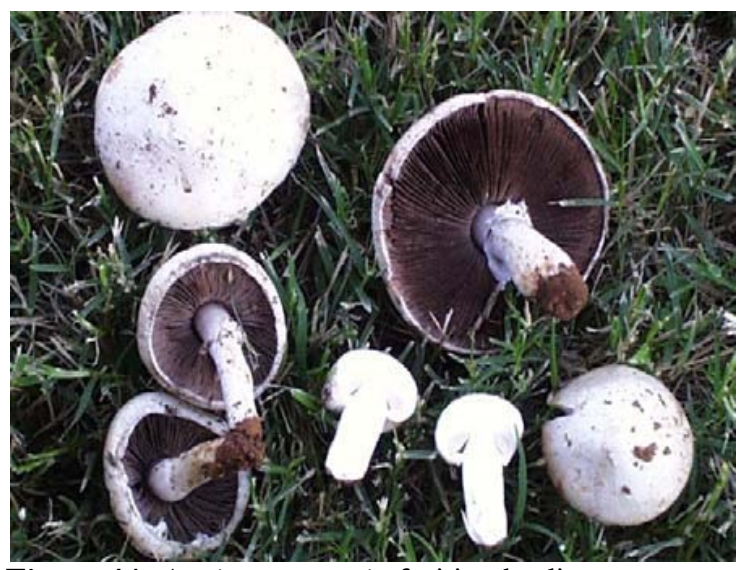

Figure 14 Agaricus campestris, fruiting bodies.

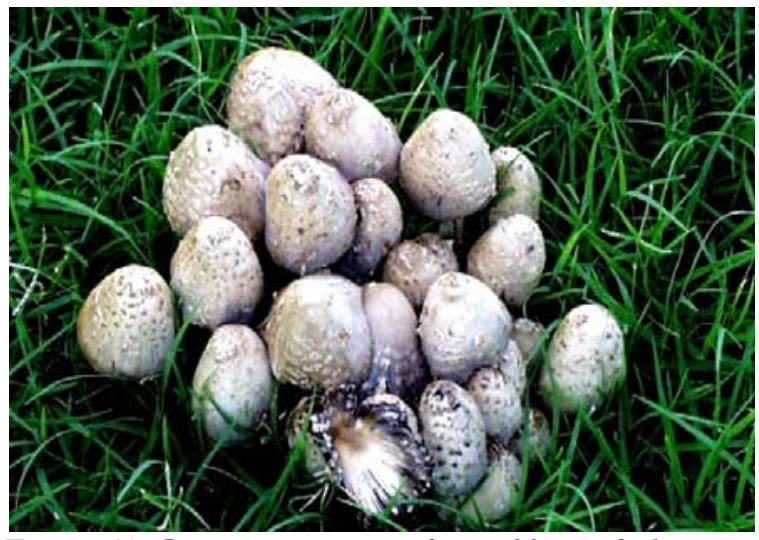

Figure 10 Coprinopsis variegata, cluster of fruiting bodies.

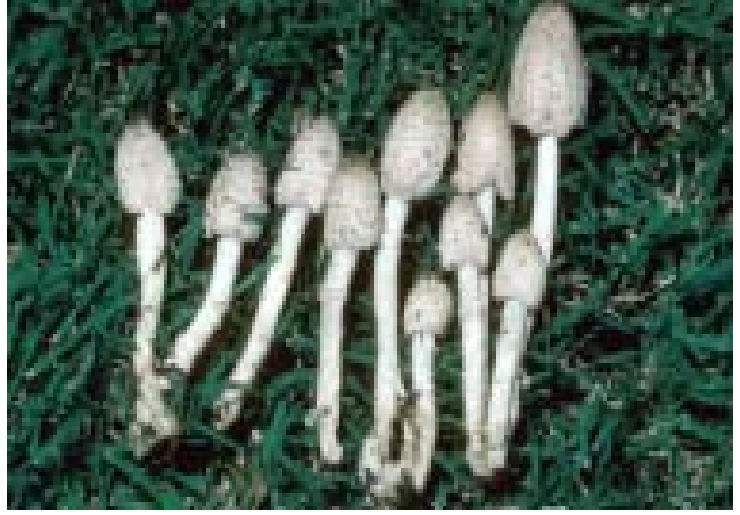

Figure 11 Coprinopsis variegata, fruiting bodies.

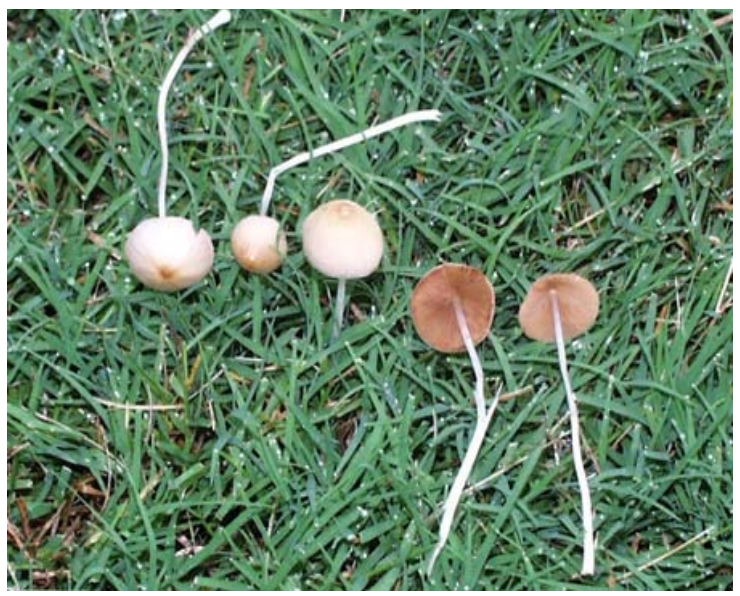

Figure 12 Conocybe lactea, fruiting bodies.

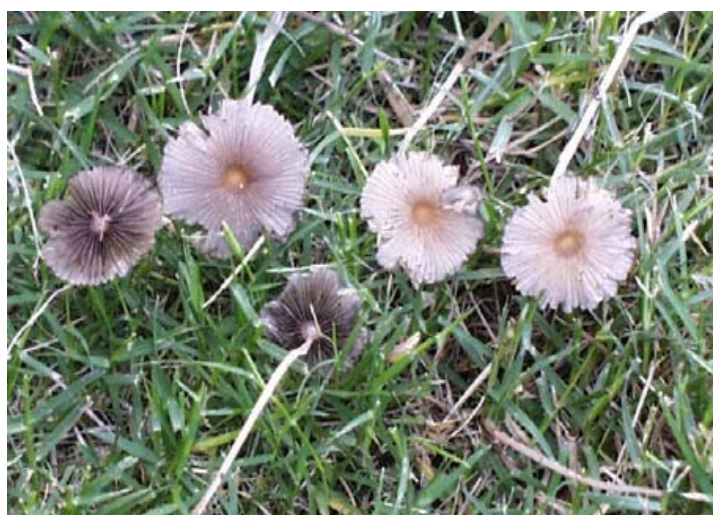

Figure 13 Parasola plicatilis, fruiting bodies.

Ovrebo, C.L. 


\section{Leucoagaricus naucinus}

Scattered on lawns, fall. Leucoagaricus naucinus is a medium-sized mushroom and is creamy white overall. The pileus is smooth, the lamellae are free and the stipe has an annulus (Figure 15). The spore print is white. This species is not edible and causes mild to severe gastric upset. There is no cup at the base as with Amanita virosa, which is similar in coloration but occurs in forests and is deadly poisonous.

\section{Pluteus petasatus}

On dead wood or wood chips, in clusters of two or three, summer. The pileus of Pluteus petasatus is off-white to light tan with the center becoming brown. The center of the pileus develops cracks in age. The lamellae are pinkish tan and free (Figure 16). The spore print is pinkish tan.

\section{Clitocybe tarda}

Scattered, on lawns, generally in clusters, fall. Clitocybe tarda is characterized by the smooth, violet-purple pileus (Figure 17). The lamellae and stipe are pigmented similarly but generally lighter. With age considerable fading of the pileus may occur. The spore print is very pale pinkish buff.

\section{Suillus brevipes}

Scattered, on soil or lawns underneath Pinus spp., late fall. Suillus brevipes has tubes and pores rather than lamellae. The pileus is brown and slimy when fresh and in age the color fades to yellowish tan and the surface may dry. The tubes and pores are yellow when young and become more olive-tinged in age (Figure 18). Suillus brevipes forms a mycorrhizal association with Pinus.

Ovrebo, C.L.

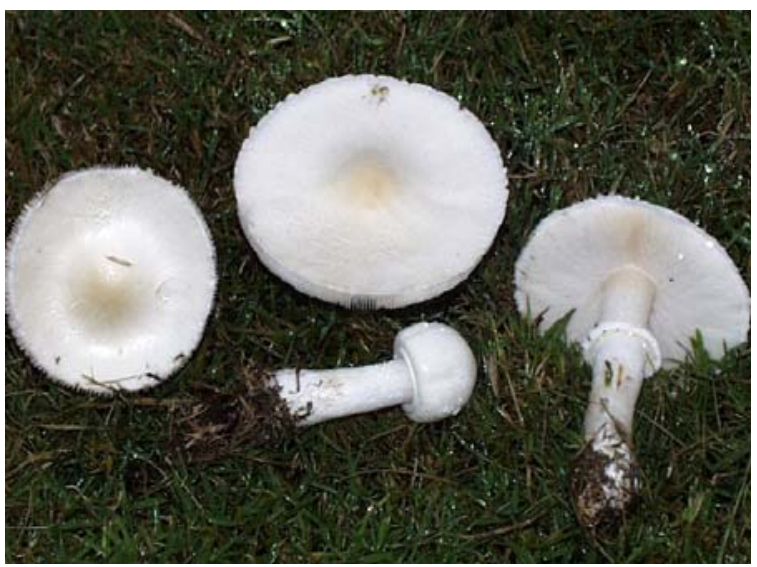

Figure 15 Lencoagaricus naucinus, fruiting bodies.

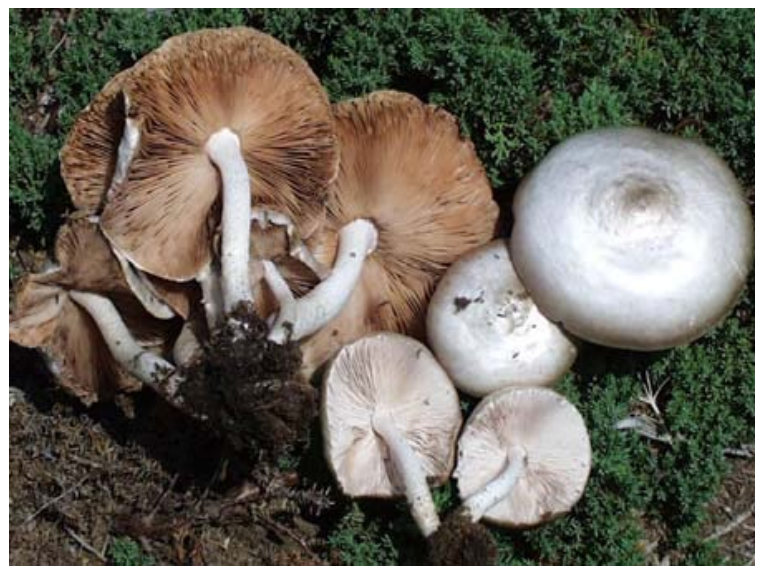

Figure 16 Pluteus petasatus, fruiting bodies.

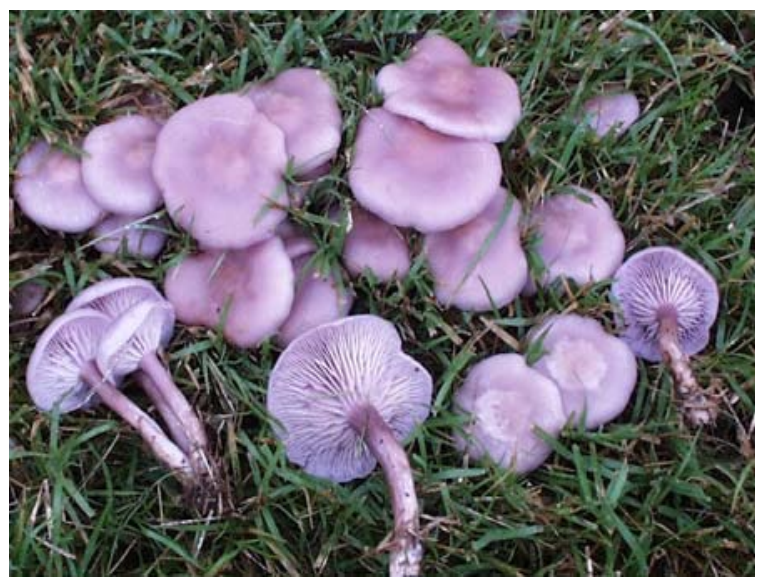

Figure 17 Clitocybe tarda, fruiting bodies.

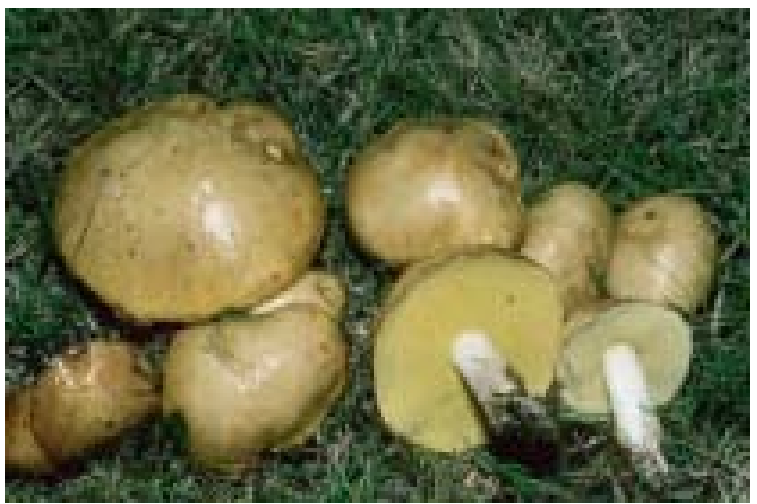

Figure 18 Suillus brevipes, fruiting bodies. 


\section{Order Phallales (Stinkhorns)}

The stinkhorns comprise a most interesting group of fleshy fungi. Rather than using air currents for spore dispersal, the spores are borne on a sticky mass called the gleba, which has a repulsive odor. For the species discussed here, the gleba is formed at the tip of the mushroom and is dark olive to nearly black. The odor attracts flies and other insects which are the agents of spore dispersal. The fungi start out as "buttons" completely encapsulated by an outer membrane (universal veil). When the mushroom bursts out of the button, a cup (volva) is left at the base. A section through the button reveals the immature fruiting body. All species of Phallus have a phallus-shaped fruiting body. Various stages of development can be seen in Figures 19-21, 23.

\section{Phallus ravenelii}

Scattered on lawns, soil or wood mulch, summer to fall. This species is identical in stature to Phallus hadriani, but the gleba is light gray, nearly smooth, and not pitted. Note the flies on the gleba of Figure 21.

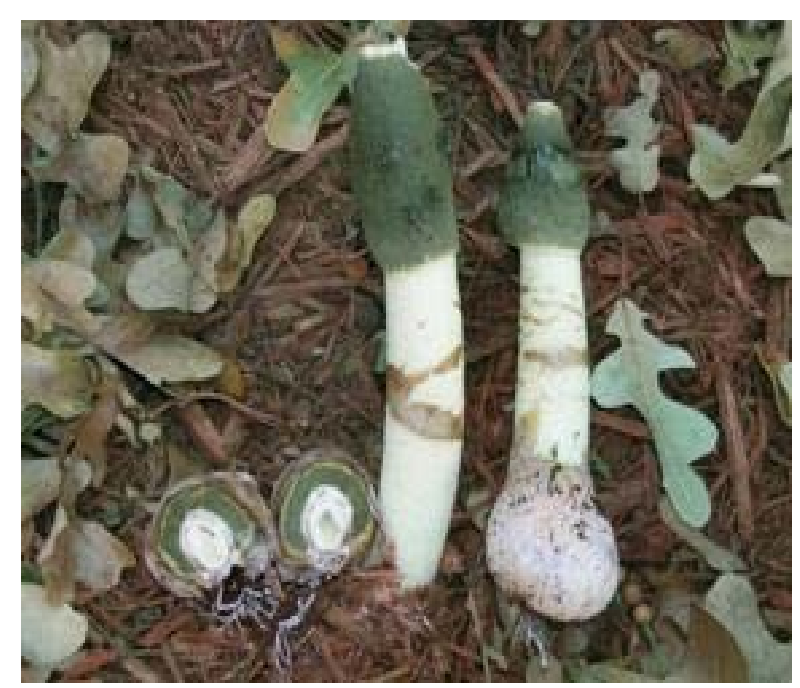

Figure 21 Phallus ravenelii, longitudinally sectioned button and fruiting bodies.

\section{Phallus hadriani}

Scattered, on lawns, soil or wood mulch, summer to fall. Phallus hadriani is distinguished by the pitted gleba (Figure 19). The surface of the volva is pink colored in this species. Phallus impudicus is identical in appearance but the outer surface of the volva is white.

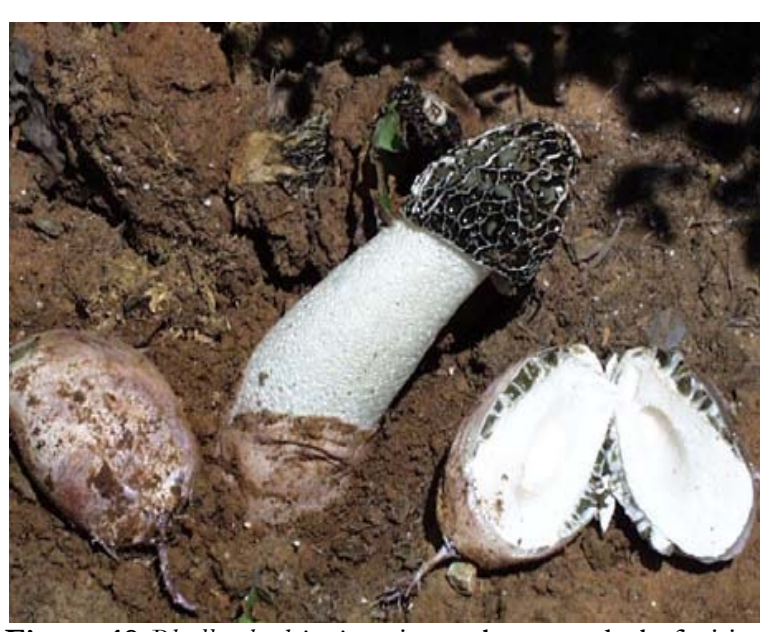

Figure 19 Phallus hadriani, an intact button whole fruiting body, and a longitudinally sectioned button.

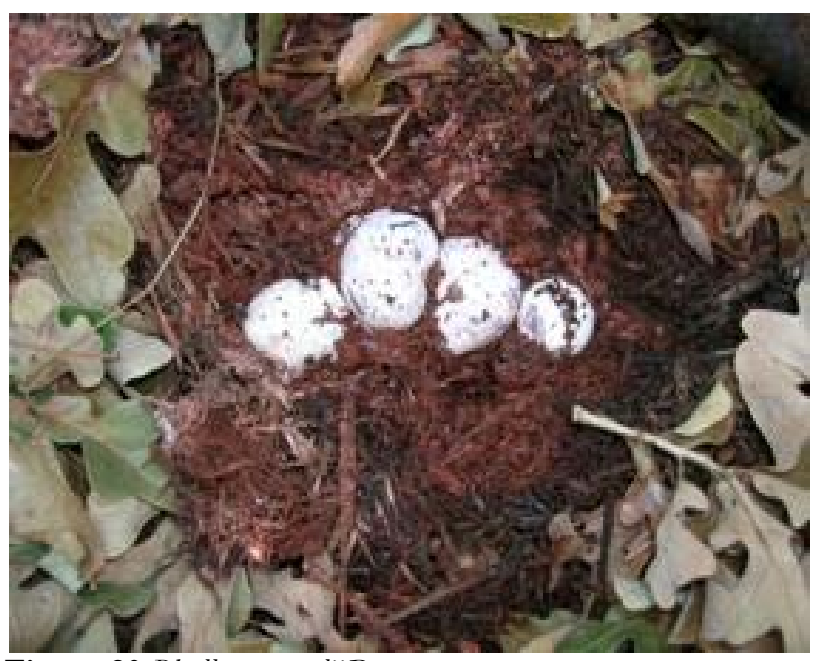

Figure 20 Phallus ravenelii,Buttons

Ovrebo, C.L. 


\section{Phallus rubicundus}

Scattered on lawns, summer. Phallus rubicundus also has the phalloid stature but the stipe is orange (Figure 22).

\section{Lysurus periphragmoides}

Solitary or scattered on lawns, summer to late fall. Lysurus periphragmoides is another common stinkhorn. It is generally smaller than the Phallus species, has an orange stipe and the glebal head is different. The glebal head has a sterile orange lattice network with gleba in between the netted pattern (Figure 23). Some field guides refer to this species as Simblum sphaerocephalum.

\section{FIELD GUIDES}

Arora, David. 1986. Mushrooms

demystified. $2^{\text {nd }}$ ed. Ten Speed Press, Berkeley, CA.

Bessette, Alan E., David W. Fischer, and A. R. Bessette. 1997. Mushrooms of Northeastern North America. Syracuse University Press.

Horn, B., R. Kay, and D. Abel. 1993. A guide to Kansas mushrooms. University of Kansas Press.

Lincoff, Gary. 1981. Audubon field guide to North American mushrooms. Alfred Knopf, New York.

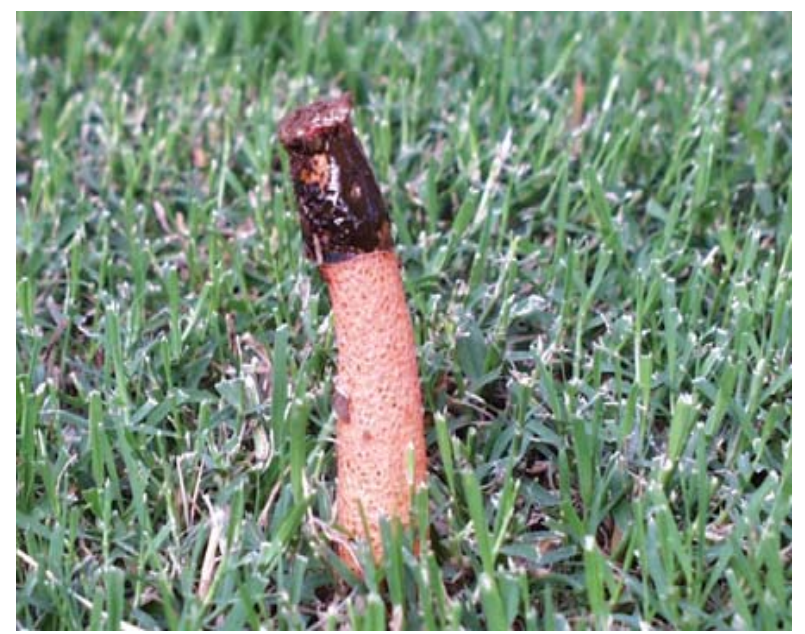

Figure 22 Phallus rubicundus, fruiting body.

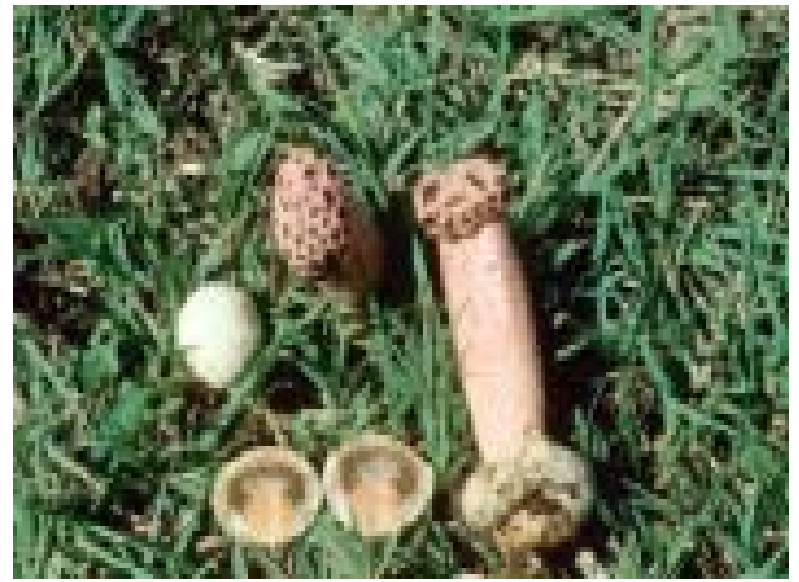

Figure 23 Lysurus periphragmoides, mature fruiting body with intact and longitudinally sectioned buttons.

Metzler, S., and V. Metzler. 1992. Texas Mushrooms. University of Texas Press, Austin.

Miller, O.K. 1977. Mushrooms of North America. Paperback ed. Dutton Publications. New York [out of print].

Smith, A. H., and Nancy S. Weber. 1981. The mushroom hunter's field guide. All colored revised ed. University of Michigan Press, Ann Arbor.

Weber, N. S., and A. H. Smith. 1985. A field guide to southern mushrooms. University of Michigan Press, Ann Arbor

Ovrebo, C.L. 